\title{
A bio-generated Fe(III)-binding exopolysaccharide used as new catalyst for phenol hydroxylation
}

\author{
Franco Baldi, ${ }^{* a}$ Davide Marchetto, ${ }^{a}$ Davide Zanchettin, ${ }^{a}$ Elisabetta Sartorato, ${ }^{b}$ Stefano Paganelli ${ }^{b}$ and \\ Oreste Piccolo $* b, c$
}

\author{
Received 8th April 2010, Accepted 14th June 2010 \\ First published as an Advance Article on the web 13th July 2010 \\ DOI: 10.1039/c004967k
}

The hydroxylation of phenol with aqueous $\mathrm{H}_{2} \mathrm{O}_{2}$ to afford catechol and hydroquinone was studied in a biphasic reaction medium, as well as in pure water, in the presence of a bio-generated iron(III) catalyst. This catalyst was purified from a culture of Klebsiella oxytoca BAS-10 growing under anaerobic conditions, with Fe(III)-citrate as the energy and carbon source. The overproduction of an exopolymer (EPS) encrusted bacterial cells. The EPS, binding $\mathrm{Fe}^{3+}$, (Fe-EPS), was extracted and studied before and after a reaction with phenol. Some of the reaction's parameters, such as temperature, $\mathrm{pH}$, and molar ratio between reagents and catalyst, were investigated to identify the best compromise between conversion and selectivity. The results could be useful either from a synthetic point of view or to support the biodegradation of aromatic substrates.

\section{Introduction}

The transition metal-catalyzed oxidation of organic substrates remains an important topic in synthetic, industrial and biological chemistry. One fundamental target is the selective hydroxylation of aromatic compounds to obtain useful multiton commodities. Furthermore, hydroxylation is often the first step to permit the degradation of toxic aromatic compounds when remediation treatments are applied.

There are numerous papers related to the iron-catalyzed hydroxylation of aromatic substrates, such as benzene and phenol, using iron compounds that are free or bound to inorganic supports, or are present in homogeneous complexes. ${ }^{1-14}$

The comparison of heme and non-heme iron enzymes that are able to catalyze a number of fascinating biological oxidations is a source of inspiration for researchers in their efforts to discover new and efficient catalytic systems. These proposed synthetic iron catalysts often contain nitrogen ligands. ${ }^{2,11-14}$ If we exclude natural ferritin, ${ }^{12}$ these nitrogen ligands have to be prepared, and may be expensive and/or not suitable for treating waste water in an environmental friendly way. In order to find new ligands for similar catalytic reactions, interest could turn to bacteria as a source of useful molecules. Several years ago, a strain of Klebsiella oxytoca BAS-10 was isolated from the acid mine drainage of pyrite mines. ${ }^{15}$ This strain produces an exopolysaccharide (EPS) during citrate fermentation, and this tricarboxylic acid is transported into the cell through a sodiumdependent carrier coupled to proton efflux by producing acetate at the end of fermentation. ${ }^{16} \mathrm{~K}$. oxytoca strain BAS-10 also

a Department of Environmental Sciences, $\mathrm{Ca}^{\prime}$ Foscari University, Calle larga Santa Marta 2137,30123, Venice, Italy.E-mail: baldi@unive.it; Tel: +39 041-2348901

${ }^{b}$ Department of Chemistry, Ca' Foscari University, Calle larga Santa Marta 2137, 30123, Venice, Italy

'SCSOP, Via Bornò 5, 23896, Sirtori, Italy.E-mail: orestepiccolo@, tin.it; Tel: +39 335-6828222 grows in $50 \mathrm{mM}$ ferric citrate and produces a thick Fe(III) hydrogel, $\mathrm{CO}_{2}$ and a solution containing acetic acid and soluble $\mathrm{Fe}(\mathrm{II})$ ions during the fermentation. ${ }^{15,17}$ Recently, a structural analysis was undertaken and the heptameric repeating unit of this Fe(III)-binding exopolysaccharide (Fe-EPS) characterized by spectroscopic methods. ${ }^{18}$ It was found that the stability formation constant of $\mathrm{Fe}^{3+}$ with one repeating unit (heptamer) of EPS was about one or two orders of magnitude larger than that of the Fe(III)-citrate complex $\left(K \approx 10^{12}-10^{13}\right.$ vs. $\left.K \approx 10^{11}\right)$ but much less than that of the Fe-EDTA complex $\left(K \approx 10^{24}\right) \cdot{ }^{17}$ In our opinion, the use of this bio-generated iron complex could be worthy of investigation as a catalyst; therefore, research to verify its catalytic ability in a model oxidation reaction, such as the hydroxylation of phenol with $\mathrm{H}_{2} \mathrm{O}_{2}$ (Scheme 1), was carried out.

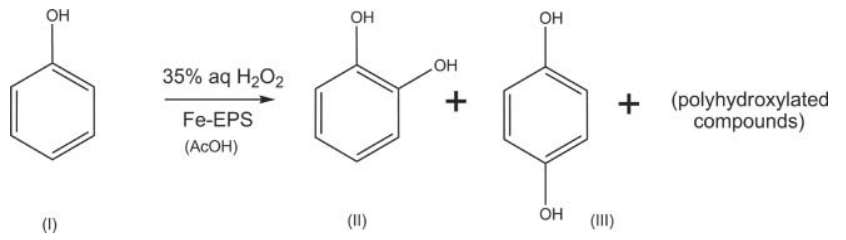

Scheme 1 The oxidation of phenol with $\mathrm{H}_{2} \mathrm{O}_{2}$ using Fe-EPS from $K$. oxytoca strain BAS- 10 as a catalyst

\section{Experimental}

\section{Cultivation of $K$. oxytoca BAS-10}

The strain was retrieved from cryovials kept at $-80{ }^{\circ} \mathrm{C}$ in $25 \%$ glycerol in nutrient broth (Difco). An aliquot of $1 \mathrm{~mL}$ of overnight culture was transferred under anaerobic conditions into two media containing, per $\mathrm{L}: 2.5 \mathrm{~g} \mathrm{NaHCO}_{3}, 1.5 \mathrm{~g} \mathrm{NH}_{4} \mathrm{Cl}$, $0.6 \mathrm{~g} \mathrm{NaH}_{2} \mathrm{PO}_{4}, 0.1 \mathrm{~g} \mathrm{KCl}$, and iron or sodium citrate species. The first medium, containing $50 \mathrm{mM} \mathrm{Fe}(\mathrm{III})$-citrate $\left(13.5 \mathrm{~g} \mathrm{~L}^{-1}\right)$, hereafter referred to as $\mathrm{FeC}$ medium, was buffered at $\mathrm{pH} 7.6$ with $\mathrm{NaOH}$. The second medium, containing $50 \mathrm{mM}\left(14.7 \mathrm{~g} \mathrm{~L}^{-1}\right)$ 
Na-citrate, hereafter referred to as $\mathrm{NaC}$ medium, was also buffered at $\mathrm{pH} 7.6$ with $\mathrm{NaOH}$.

The cultivation of strain BAS-10 was performed separately either in $1 \mathrm{~L}$ of $\mathrm{FeC}$ medium or in $1 \mathrm{~L}$ of $\mathrm{NaC}$ medium, incubated at $30{ }^{\circ} \mathrm{C}$ under anaerobic conditions. After $10 \mathrm{~d}$, the suspension was first centrifuged to eliminate bacterial cells, and then the supernatant was treated with $800 \mathrm{~mL}$ of cooled ethyl alcohol $(95 \%)$ to precipitate the polysaccharides. The purification was repeated twice. Both colloidal materials from the $\mathrm{FeC}$ and $\mathrm{NaC}$ media were dried out under vacuum to obtain either Fe-EPS or Na-EPS as solid materials.

\section{Microscope observations}

To observe the iron in EPS and cell envelope conditions, $K$. oxytoca BAS-10 cells were grown in $\mathrm{FeC}$ medium after $10 \mathrm{~d}$ of incubation at $30^{\circ} \mathrm{C}$. During cell growth, after hydrogel precipitation, several specimens were prepared for transmission electron microscopy (TEM) according to the usual procedure, cells being harvested by centrifugation at $8100 \mathrm{~g}$. The bacterial pellet was fixed for $1 \mathrm{~h}$ at $4{ }^{\circ} \mathrm{C}$ with $2.5 \%$ glutaraldehyde, after with $0.1 \mathrm{M}$ lysine in a $0.066 \mathrm{M}$ cacodylate buffer at $\mathrm{pH} 7.2$ for $30 \mathrm{~min}$ at room temperature. The cells were washed five times with the same buffer and post-fixed for $1 \mathrm{~h}$ at room temperature in $1 \%$ osmium tetroxide, rinsed with distilled water and embedded in Spurr resin. Ultrathin sections were prepared using a LKB II Nova Ultramicrotome device with a diamond knife. Sections were stained with 3.0\% uranyl acetate solution for $15 \mathrm{~min}$, washed once with distilled water and incubated in lead citrate for $10 \mathrm{~min}$. TEM observations were performed using a JEOL JEM 100b (Tokyo, Japan) instrument operating under standard conditions (Fig. 1A).

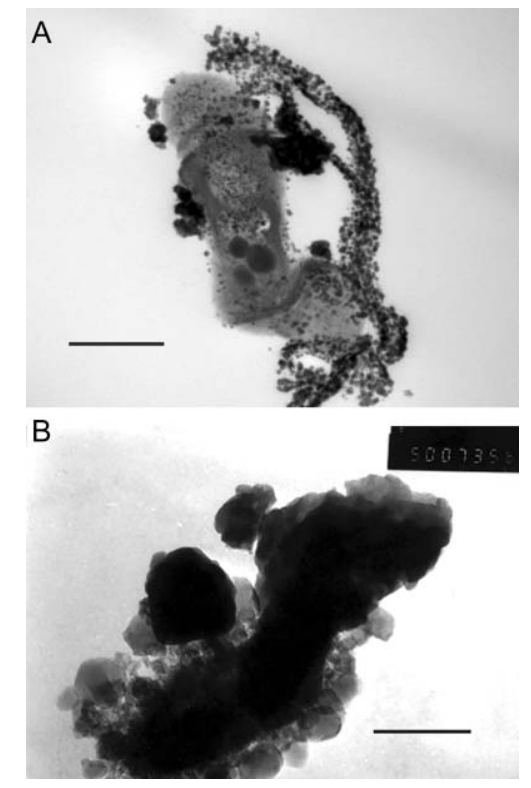

Fig. 1 A: A TEM micrograph of a BAS-10 strain producing an exopolysaccharide rich in iron after fermentation in the presence of $\mathrm{Fe}(\mathrm{III})$-citrate under anaerobic conditions $(25000 \times)$. B: An ESEM micrograph of a BAS-10 strain that is entirely encrusted with iron deposits.
A TEM analysis was also undertaken directly without a staining preparation, mounting sample on copper grids to determine the $\mathrm{Fe}$ (III)-EPS structure after the precipitates formed in the reaction vessel of the phenol transformation.

An aliquot of $10 \mu \mathrm{L}$ of ferric hydrogel was deposited and smeared on an aluminium stub in order to analyze the iron encrusted cells of the BAS-10 culture using an environmental scanning electron microscope (ESEM). Specimens were visualized by a scanning electron microscope (FEI Quanta mod. 200, Eindhoven, NL) (Fig. 1B).

\section{General procedure of the oxidation reaction}

In a $250 \mathrm{~mL}$ jacketed glass reactor, $16.9 \mathrm{mg}$ of Fe-EPS (equivalent to about $6 \mathrm{mg}$ of $\left.\mathrm{Fe}^{+3}\right),{ }^{17} 0$ or $0.4 \mathrm{mmol}$ of $\mathrm{RCOOH}$ [trifluoroacetic acid (TFA) or acetic acid (AcOH)], 0 or $12 \mathrm{~mL}$ of acetonitrile (AN) and 0 or $10 \mathrm{~mL}$ of water were stirred $2 \mathrm{~h}$ at room temperature. Next, $1.69 \mathrm{~g}(18 \mathrm{mmol})$ of phenol was added, and the mixture heated and maintained at $37^{\circ} \mathrm{C}$. Finally, an aqueous solution of $35 \%(w / v) \mathrm{H}_{2} \mathrm{O}_{2}$ was slowly added

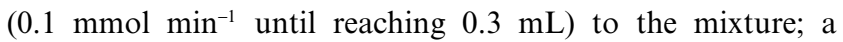
second identical quantity of $\mathrm{H}_{2} \mathrm{O}_{2}$ was added after $2 \mathrm{~h}$ (in some experiments, further quantities of this reagent were added). It was decided to stop and work-up each test after $24 \mathrm{~h}$ to be sure that all the $\mathrm{H}_{2} \mathrm{O}_{2}$ had been consumed. The mixture was acidified (to about $\mathrm{pH}$ 1), filtered on a sintered glass, extracted with $2 \times 10 \mathrm{~mL}$ of 2-methyl-tetrahydrofuran (MTF) and the organic phase analyzed by gas chromatography [GC system: Agilent 6850; HP-1 column $(30 \mathrm{~m} \times 0.32 \mathrm{~mm} \times 0.25 \mathrm{~mm}) ; T=$ $80{ }^{\circ} \mathrm{C}(5 \mathrm{~min})$ then up to $250{ }^{\circ} \mathrm{C}$ at $10^{\circ} \mathrm{min}^{-1}$. The results of the different runs under each set of reaction conditions are reported in Table 1. The conversion of phenol and the amount of the dihydroxylated products (catechol and hydroquinone) was determined by calibration $v$ s. cyclooctane, taken as an internal standard for the GC analyses.

\section{SEM micrographs}

SEM micrographs were taken to investigate the structural properties of the precipitate obtained from the treatment with phenol and $\mathrm{H}_{2} \mathrm{O}_{2}$ in pure water, and the images were analyzed to verify, in particular, the emergence of recurrent patterns resembling ordered particles by the Fourier decomposition of selected lines of the raster grayscale. Practically, each line provided a sequence of pixels, each with a value ranging from 0 (black: electron dense iron) to 255 (white: space between particles), which were then processed analogously to a time series. It is notable that the three sections cut the image with the same angle and preserved the image's original dimensions (i.e., an isomeric rotation was performed). Spectral densities were evaluated by smoothing the periodograms with a $w=5$ Hamming window. As for temporal series, data autocorrelation was used to evaluate the background noise spectrum, i.e., the energy that is likely to be produced at a given scale by a random noise process. Accordingly, only spectral peaks that were significantly above the background noise spectrum were assumed to be a true feature of the investigated process. In particular, sample s1 was used to deduce the properties of the background noise spectrum by treating the grayscale sequence as a first order autoregressive process, i.e., according to the formula ${ }^{19}$ 
Table 1 Experimental conditions and results of phenol oxidation by $\mathrm{H}_{2} \mathrm{O}_{2}$ in the presence of a catalytic amount of Fe-ESP

\begin{tabular}{|c|c|c|c|c|c|c|}
\hline Run & Solvent(s) $(/ \mathrm{mL})^{a}$ & $\mathrm{RCOOH}(/ \mathrm{mmol})^{a}$ & $\mathrm{H}_{2} \mathrm{O}_{2} / \mathrm{mL}[/ \mathrm{mmol}]$ & Conversion $(\%)$ & Catechol (II) (\%) & Hydroquinone (III) (\%) \\
\hline 2 & $\mathrm{H}_{2} \mathrm{O}(10)$ & TFA $(0.4)$ & $1.3[14.7]$ & 10 & 7 & 3 \\
\hline 4 & $\mathrm{AN} / \mathrm{H}_{2} \mathrm{O}(12 / 10)$ & $\mathrm{AcOH}(0.4)$ & $1[11.3]$ & 25 & 17 & 8 \\
\hline 5 & $\mathrm{AN} / \mathrm{H}_{2} \mathrm{O}(12 / 10)$ & $\mathrm{AcOH}(0.4)$ & $0.6[6.8]$ & 22 & 15 & 7 \\
\hline $6^{b}$ & $\mathrm{AN} / \mathrm{H}_{2} \mathrm{O}(12 / 10)$ & $\mathrm{AcOH}(0.8)$ & $1.2[13.6]$ & 18.3 & 9.5 & 4.9 \\
\hline 7 & $\mathrm{H}_{2} \mathrm{O}(10)$ & - & $0.6[6.8]$ & 20 & 14 & 6 \\
\hline
\end{tabular}

${ }^{a} \mathrm{AN}=$ acetonitrile, TFA $=$ trifluoroacetic acid, $\mathrm{AcOH}=$ acetic acid, $\mathrm{MTF}=2$-methyl- tetrahydrofuran. ${ }^{b}$ In these experiments, the quantities of phenol [3.38 $\mathrm{g}(36 \mathrm{mmol})]$ and Fe-EPS [33.8 $\mathrm{mg}$, corresponding to about $12 \mathrm{mg}$ of $\left.\mathrm{Fe}^{+3}\right]$ were doubled in comparison with the other runs.

$$
F(\mathrm{w})=\left(1-\alpha^{2}\right) /\left(1+\alpha^{2}-2 \alpha \cos (2 \pi w / N)\right),
$$

where $\alpha$ is the sample s1 lag-1 autocorrelation, $w=1 \ldots N / 2$ is the frequency index in the spatial domain and $N$ is the number of points of the sample (pixels). The significance (set at the $5 \%$ level in this study) was calculated ${ }^{20}$ by multiplying the background spectrum by the 95 th percentile value for a chi-square distribution $\chi^{2}$.

Three sequences were obtained from the images of SEM micrographs (Fig. 2A), representing, respectively, an outer unorganized state (series s1), an amorphous state (series s2) and an ordered state (series s3) (Fig. 2B).

\section{FT-IR analysis}

During the reaction of phenol with $\mathrm{H}_{2} \mathrm{O}_{2}$ in pure water, as reported above, a precipitate was observed. To exclude the formation of ferric oxides, this precipitate, as well as Na-EPS (produced in $\mathrm{NaC}$ medium) and Fe-EPS (produced in $\mathrm{FeC}$ medium and used as a catalyst in this reaction), were analyzed by FT-IR. The IR spectra ( $\mathrm{KBr}$ pellets) were recorded on an FT-IR Nicolet Magna 750 instrument (Fig. 3).

\section{Results and discussion}

In previous reports ${ }^{8,9}$ it was demonstrated that strain BAS-10 produces, under anaerobic conditions, a polysaccharide with a heptameric repeating structure with four $\alpha$-rhamnose, two $\beta$ glucuronic acids and one $\beta$-galactose as follows:

$2)-\alpha-$ Rha- $(1 \rightarrow 3)-\beta$-Gal- $(1 \rightarrow 2)-\alpha-$ Rha- $(1 \rightarrow 4)-\beta$-GlcA- $(1 \rightarrow 3)-\alpha-$ Rha- $(1 \rightarrow 3)-\alpha-$ Rha- $(1 \rightarrow$
4
$\stackrel{4}{\wedge}$
$\beta-$ GlcA

Each heptameric unit is able to bind $\mathrm{Fe}^{3+}$ up to $36 \pm 4.5 \%$. The production of total Fe-EPS is about $6.65 \mathrm{~g} \mathrm{~L}^{-1}$ (on dry weight) in FeC medium and that of $\mathrm{Na}$-EPS is only $0.7 \mathrm{~g} \mathrm{~L}^{-1}$ in $\mathrm{NaC}$ medium. BAS-10 cells produce this $\mathrm{Fe}(\mathrm{III})$ complex very late in the stationary phase when the medium is spent. Cells, observed by TEM, are surrounded by a dense EPS structure with spherical electrodense particles in the row. As seen by ESEM observations, a cell is completely encrusted by ultradense particles containing iron (Fig. 1B).

When Fe-EPS was used as a catalyst in pure water, after $3 \mathrm{~h}$ of contact with phenol and $\mathrm{H}_{2} \mathrm{O}_{2}$, a precipitate was observed. This material was analyzed to find out if iron was freed
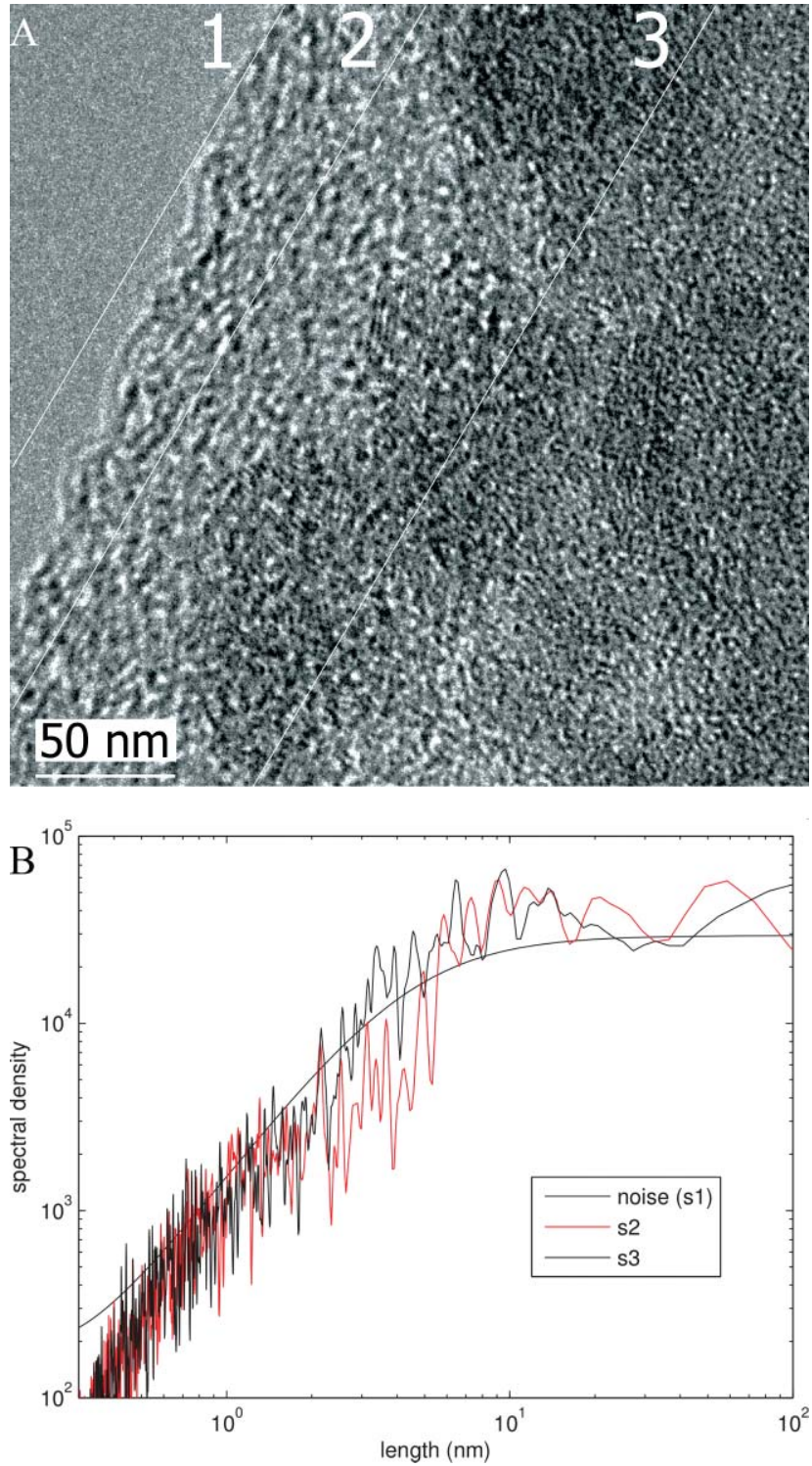

Fig. 2 A: SEM micrograph of purified precipitated Fe polysaccharide obtained in water during the oxidation reaction. B: Spectral densities of samples s2 (amorphous state) and s3 (ordered state) compared to the $95 \%$ confidence level of the theoretical background noise spectrum estimated from sample s1 (outer unorganized state). 


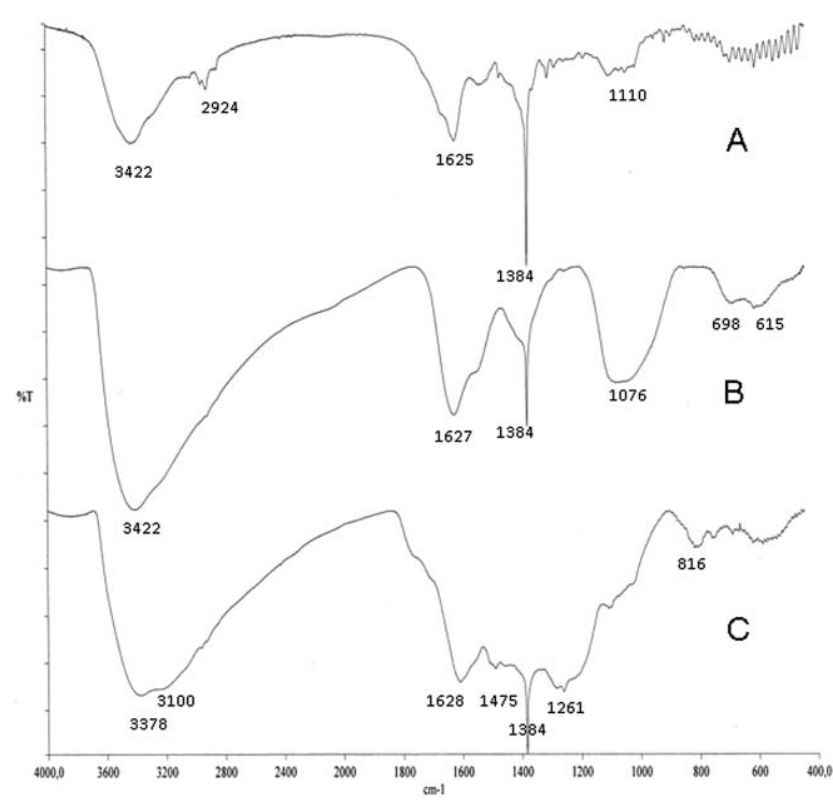

Fig. 3 FTIR spectra of A: purified Na-ESP from $\mathrm{NaC}$ medium, B: purified $\mathrm{Fe}-\mathrm{ESP}$ from $\mathrm{FeC}$ medium and $\mathrm{C}$ : precipitated $\mathrm{Fe}$ polysaccharide.

from the EPS and transformed in oxides during the reaction. An X-ray diffractometry determination did not seem to show the presence of iron crystals. Also, FT-IR confirmed that iron in this precipitate was still bound to EPS, but contained additional bands, very probably due to aromatic compounds, such as phenol and its hydroxylation products (Fig. 3). The pronounced shoulder at $3100 \mathrm{~cm}^{-1}$ is assigned to the aromatic $\mathrm{C}-\mathrm{H}$ stretching, while the bands at $1500-1450 \mathrm{~cm}^{-1}$ may be due to $\mathrm{C}=\mathrm{C}$ stretching vibrations. The peaks in the region $1300-1200 \mathrm{~cm}^{-1}$ are supposed to correspond to the aromatic $\mathrm{C}-\mathrm{O}$ stretching and to the ring hydrogen rocking vibration, respectively. Moreover, aromatic substitution bands are evident in the region between 850 and $650 \mathrm{~cm}^{-1}$. Therefore, the precipitate might be a different, less soluble Fe-EPS conformer that includes some molecules of the phenol derivatives or a modified iron polysaccharide complex, where some molecules of the phenol derivatives are present as new ligands of the metal; the latter would exhibit a lower solubility in the aqueous medium in the absence of an organic solvent. Actually, it is not possible to distinguish between these two possibilities. Analysis of the SEM image of this precipitate (Fig. 2B) shows the density spectrum of s2 (amorphous state) and s3 (ordered state), and a comparison of them with the background noise spectrum deduced by s1 (unorganized state). Spectral peaks above the $95 \%$ confidence level for the background noise separate out the true features of the series, i.e., in this case, the structural properties of the observed material that do not originate by chance. The two series show similar properties at very small scales, i.e., $<1 \mathrm{~nm}$, also with comparable signals at 2 and $9-15 \mathrm{~nm}$, therefore pointing to nanostructures of such scales being present in both the amorphous and ordered material. Conversely, an easily identifiable structural organization emerges in series $\mathrm{s} 3$ around the $2.5-5 \mathrm{~nm}$ band, as well as around $6.5 \mathrm{~nm}$, while series s2 shows a low structural organization at 5.5 and $7.2 \mathrm{~nm}$ scales. The relevance of these findings emerges as far as these are drawn back to the possible properties of the ordered structures in iron polysaccharide after precipitation.

The more relevant results of using Fe-EPS as a catalyst in the hydroxylation of phenol are reported in Table 1. Each test, except for 6 and 8, was duplicated, and the results represent an average of two runs. Using an accurate calibration method vs. a standard for GC analyses, the mass balance of compounds (I), (II) and (III) in experiments 6 and 8 corresponded to $95-96 \%$, so indicating that no polyhydroxylated products were present in a relevant amount.

To single out the more important factors affecting the conversion and selectivity, experiments were carried out to change the amount and nature of the solvents and/or of $\mathrm{RCOOH}$, and the ratio between phenol and $\mathrm{H}_{2} \mathrm{O}_{2}$. It was also observed that a dried, aged Fe-EPS sample showed lower or no catalytic activity in this reaction, but the activity was recovered after pulverization of the solid and its rehydration in water. In the absence of water, the polymeric structure probably reorganizes itself so that $\mathrm{Fe}^{3+}$ becomes unavailable to the other reagents. On the basis of these results, it is possible to see that the reaction occurs at a neutral $\mathrm{pH}$ as well as when an organic acid of moderate acidity is present, with a slight better performance in the latter case. The use of TFA, a preferentially added acid according to the literature, is, on the contrary, deleterious. No reaction occurs when an organic solvent that is not miscible with water is present, probably because, in this case, phenol prefers to stay in the organic phase. For this aromatic substrate, a solvent such as acetonitrile is probably useless, but for other less polar aromatic substrates, the presence of a water-miscible organic solvent might help contact be made between the substrate and the catalyst. The molar ratio between the dihydroxylated products, catechol (II) and hydroquinone (III), is about 2, but there is a slight difference when working in a more or less concentrated mixture (compare experiments 7 and 8 , where a ratio of 2.3 vs. 1.7 was measured). A small quantity of catechol might remain preferentially entrapped into the catalyst as a new metal ligand, so decreasing the value of this ratio when the mixture is less dilute. As a matter of fact, the analytical results of these experiments before acidification of the reaction mixture up to $\mathrm{pH} 1$ showed, in general, a lower total yield of dihydroxylated products and a worse mass balance.

On the basis of the added quantity of $\mathrm{H}_{2} \mathrm{O}_{2}$, it is possible to calculate that the amount of $\mathrm{H}_{2} \mathrm{O}_{2}$ actually used for the reaction is $48-58 \%$, while the remaining amount is decomposed. On the contrary, the reacted phenol is nearly quantitatively converted into catechol and hydroquinone (i.e. the selectivity of phenol is $\geq 95 \%$ ). These results are encouraging and might be, in our opinion, further improved by scaling it up and fine tuning the addition rate of $\mathrm{H}_{2} \mathrm{O}_{2}$. Finally, in one experiment, the reaction temperature was increased to $55{ }^{\circ} \mathrm{C}$, but without any change in the results. In a blank experiment where Na-EPS was used instead of Fe-EPS, no reaction was observed, as expected.

\section{Conclusions}

Although detailed mechanistic information is not currently available, the results suggest that this oxidation reaction of phenol in the presence of Fe-EPS could proceed by two parallel mechanisms, and not by a pure radical mechanism, where ${ }^{\circ} \mathrm{OOH}$ and/or ${ }^{\circ} \mathrm{OH}$ radicals are present and act as oxidizing 
agents, as in the Fenton reaction. In fact, the high selectivity of phenol suggests the presence of transient species that are more sterically demanding than the simple hydroxyl radical, as suggested previously. ${ }^{2,13}$

This new bio-generated Fe(III) catalyst, working under optimal reaction conditions, compares quite well, in our opinion, to other Fe-based catalytic systems considered state-of-theart, ${ }^{3,5,6,11,12,14,21}$ with some advantages in terms of cost and low environmental impact.

\section{Acknowledgements}

The authors wish to thank Mr Mattia Amadio for certain catalytic experiments, Dr Patrizia Canton for the SEM analyses and Prof. Paola Zuddas of the University of Cagliari for ESEM analyses of the bacteria cells.

\section{References}

1 M. B. Hocking and D. J. Intihar, J. Chem. Technol. Biotechnol. A, $1985, \mathbf{3 5}, 365$

2 J. P. Hage, A. Llobet and D. T. Sawyer, Bioorg. Med. Chem., 1995, 3, 1383.

3 D Wang, Z. Liu, F. Liu, X. Ai, X. Zhang, Y. Cao, J. Yu, T. Wu, Y. Bai, T. Li and X. Tang, Appl. Catal., A, 1998, 174, 25.

$4 \mathrm{~J}$ Guo and M. Al-Dahhan, Ind. Eng. Chem. Res., 2003, 42, 2450.

5 A. L. Villa, C. A. Caro and C. Montes de Correa, J. Mol. Catal. A. Chem., 2005, 228, 233.
6 J-S. Choi, S-S. Yoon, S-H. Jang and W-S. Ahn, Catal. Today, 2006, 111, 280.

7 M Kurian and S. Sugunan, Chem. Eng. J., 2006, 115, 139.

8 J. Peng, F. Shi, Y. Gu and Y. Deng,, Green Chem., 2003, 5, 224.

9 Y. Li, H. Xia, F. Fan, Z. Feng, R. A. van Santen, E. J. M. Hensen and C. Li, Chem. Commun., 2008, 774.

10 H Xin, A. Koekkoek, Q. Yang, R. A. van Santen, C. Li and E. J. M. Hensen, Chem. Commun., 2009, 7590.

11 C Liu, X. Ye, R. Zhan and Y. Wu, J. Mol. Catal. A: Chem., 1996, 112,15 .

12 N. Zhang, F. Li, Q. J. Fu and S. C. Tsang, React. Kinet. Catal. Lett., 2000, 71, 393.

13 D Bianchi, M. Bertoli, R. Tassinari, M. Ricci and R. Vignola, J. Mol. Catal. A: Chem., 2003, 204-205, 419.

14 H. S. Abbo, S. J. J. Titinchi, R. Prasad and S. Chand, J. Mol. Catal. A: Chem., 2005, 225, 225.

15 F Baldi, A. Minacci, M. Pepi and A. Scozzafava, FEMS Microbiol. Ecol., 2001, 36, 169.

16 J. S. Lolkema, H. Enequist and M. E. Van Der Rest, Eur. J. Biochem., 1994, 220, 469.

17 F. Baldi, D. Marchetto, D. Battistel, S. Daniele, C. Faleri, C. De Catro and R. Lanzetta, J. Appl. Microbiol., 2009, 107, 1241.

18 S. Leone, C. De Castro, M. Parrilli, F. Baldi and R. Lanzetta, Eur. J. Org. Chem., 2007, 5183.

19 D. B. Percival and A. T. Walden, Spectral Analysis for Physical Applications, 1993 Cambridge University Press, Cambridge.

20 D. Zanchettin, A. Rubino, P. Traverso and M. Tomasino, J. Geophys. Res., 2008, 113, D12102.

21 K. M. Parida, S. Singha and P. C. Sahoo, Catal. Lett., 2010, 136, 155 (published after the completion of our work). 